http://ensaios.usf.edu.br/

\title{
TERAPIA POR CONTENSÃO INDUZIDA EM PACIENTE COM AVC INFANTIL: ESTUDO DE CASO \\ CONSTRAINT-INDUCED MOVEMENT THERAPY IN PRESCHOOL CHILDREN WITH PEDIATRIC STROKE: A CASE STUDY
}

OLIVEIRA, Carolina Camargo de ${ }^{1}$; TOVAZI, Luma Fernanda de Azevedo ${ }^{2}$; NEVES, Milena Catarina Romero das Neves ${ }^{3}$

\section{carolina.oliveira@usf.edu.br}

RESUMO. O objetivo deste estudo foi valiar os efeitos da Terapia por Contensão Induzida (TCI) na função manual e amplitude de movimento (ADM) do membro superior parético (MSP) de criança com AVC. Trata-se de estudo de caso com paciente hemiparético à D, avaliado com o Pediatric Motor Activity Log (PMAL) e Goniometria do MSP, sendo realizada uma avaliação inicial (AI); avaliação final I (AFI) ao final da intervenção e avaliação final II (AFII) três meses após. A TCI teve duração de um mês, três dias por semana com duração de três horas cada, sendo estimulado a realizar exercícios fisioterapêuticos, atividades de vida diária, tarefas de lazer e escolares com o MSP. A AI foi comparada com a AFI em que pode-se observar aumento na pontuação principalmente em qualidade e, na comparação da AFI com a AFII, houve melhora principalmente na frequência, além de manutenção dos ganhos obtidos na AFI. Quanto à Goniometria, houve melhora principalmente da ADM ativa de alguns movimentos do membro e pouca diminuição de alguns movimentos na AFII. A TCI mostrou-se eficaz para melhora da função manual e na ADM do MSP em paciente com AVC infantil, podendo servir como base para estudos com amostras maiores.

Palavras-chave: AVC Infantil, Paresia, Reabilitação, Terapia por Contensão Induzida.

ABSTRACT. Study objective of evaluate the effects of Therapy For Containment Induced (TCI) in the manual function and range of motion (ROM) of the paretic upper limb (PUL) in children with stroke. This is a case study with hemiparetic patient to D as measured by the Pediatric Motor Activity Log (PMAL) and Goniometry PUL, being performed an initial assessment (IA); finally assessment I (FAI) at the end of the intervention and finally evaluation II (FAII), three months after the end. TCI lasted one month, three days a week lasting three hours each, being encouraged to perform physical therapy exercises, daily activities, leisure and school tasks with the PUL. IA was compared with the FAI that can be observed increase in the score mainly in quality and, in comparison the FAI with FAII, was improved primarily in frequency, and maintaining the gains in FAI. As for Goniometry, an improvement mainly of active ROM of some movements member and others have hardly ROM decreases in FAII. TCI was effective for improvement in hand function and ROM in patient with pediatric stroke, and can serve as a basis for studies with larger samples.

Key words: Children Stroke, Paresis, Rehabilitation, Induced Containment Therapy. 


\section{INTRODUÇÃO}

O Acidente Vascular Cerebral (AVC) é definido como o desenvolvimento rápido de sintomas clínicos de perturbações focais ou globais da função cerebral com sintomas permanecendo por mais de 24 horas (MATTA; GALVÃO; et al, 2006).

De acordo com faixa etária podem ser classificados em fetal, quando o diagnóstico é feito ainda intra útero ou na primeira semana pós natal, onde estão presentes sinais imagiológicos que evidenciam perda tecidular. O AVC neonatal precoce é diagnosticado nos três primeiros dias após o nascimento, neonatal tardio entre 4 a 28 dias após o parto, perinatal presumível após 28 dias do nascimento. Os AVCs perinatais ocorrem entre as 20 semanas de gestação e os 28 dias de vida após o nascimento, e podem ser confirmados por exames de neuroimagem ou anatomia patológica. Já o AVC na infância é considerado quando ocorre entre 30 dias de vida até 18 anos de idade (RODRIGUES; MAFALDA; 2015). O acidente vascular pré-natal, que ocorre ainda intra-útero fica evidente entre o $4^{\circ}$ e $12^{\circ}$ mês de vida, quando nota-se a dominância de uma das mãos, decorrente da hemiparesia contra-lateral. Através de exames de imagem pode-se notar uma área de infarto, que confirma o diagnóstico, porém não determina quando ocorreu o acidente (DEVEBER; 2006).

O AVC infantil é raro, afetando cerca de 2 a 8 em cada 100 mil crianças, predominante no sexo masculino e gera sequelas motoras, cognitivas, auditivas, distúrbios de linguagem, epilepsia, entre outros. A sequela mais comum do AVC é a hemiparesia MEKETERIAN; CARVALHO, 2009; ROTTA; SILVA, 2002).

Seu diagnóstico pode ser difícil já que a apresentação clínica no momento agudo é, muitas vezes, sútil e a investigação radiológica precoce pode não mostrar alterações (MATTA; GALVÃO; et al, 2006; GUIMARÃES; CIASCA, 2006).

A etiologia do AVC infantil é diferente do AVC em adultos sendo as principais causas: cardiopatias congênitas, doenças hematológicas (anemia falciforme, leucemia, linfomas e outras), vasculite pós-infecciosa, anomalias vasculares, coagulopatias, infartos venosos, doenças metabólicas, vasoespasmo, traumas, causas genéticas, entre outras. As mais comuns são as causas hematológicas, genéticas e as doenças cardíacas (MEKETERIAN; CARVALHO, 2009; ROTTA; SILVA, 2002) .

Assim como em adultos, o AVC infantil pode ser hemorrágico ou isquêmico, mas devido a alta taxa de mortalidade do AVC hemorrágico, a maioria das crianças apresentam sequelas de AVC isquêmico (MATTA; GALVÃO; et al, 2006).

$\mathrm{O}$ membro superior tende a ser afetado de forma mais severa que o membro inferior gerando quadro de hemiparesia ou a hemiplegia, que pode causar impacto nas atividades de vida diária, além de prejudicar outras funções como escrita, brincadeiras, gesticulações, entre outras, que geram prejuízos funcionais a estas crianças. A Fisioterapia pode contribuir de forma eficaz para reestabelecer a função ou mesmo agir de forma a prevenir déficits motores secundários nos casos de AVC infantil (MATTA; GALVÃO; et al, 2006).

A Terapia por Contensão Induzida (TCI) ou Técnica de Restrição terapêutica tem por objetivo recuperar a função do membro superior (MS) que apresenta déficits motores após lesões encefálicas (SILVA; TAMASHIRO, 2010; GIANLORENCO; KIRISAWA, 2013).

O foco deste programa terapêutico é a desprogramação do desuso motor, ou seja, fazer com que o paciente hemiparético volte a utilizar o MS acometido após a lesão (GAMBA; CRUZ, 2011).

Durante as sessões, o paciente tem o MS não afetado imobilizado por uma luva ou tipóia e realiza a prática de movimentos funcionais. Palavro e Schuster (MATTA; GALVÃO; et al, 2006) ${ }^{1}$. constatataram que diferentes protocolos com durações diversas com relação ao tempo de contensão do membro superior e com relação à duração total do tempo de terapia, resultaram em efeitos positivos gerando benefícios motores e otimizando a função manual do membro parético em todos os casos independente do protocolo aplicado. 
Após a análise de diversos estudos recentes, foi observado por meio de exames de neuroimagem que o estímulo do uso forçado do MS acometido durante essa forma de terapia gerou estimulação da área encefálica afetada (PALAVRO; SCHUSTER, 2013).

O objetivo deste estudo foi avaliar os efeitos da TCI na função manual e na amplitude de movimento (ADM) no membro superior parético de uma criança com AVC.

\section{METODOLOGIA}

Trata-se de estudo de caso, aprovado pelo Comitê de Ética em Pesquisa da Universidade São Francisco (Parecer No: 1.245.114/2015). Foram rastreados pacientes com idades entre 2 a 8 anos, com diagnóstico clínico confirmado de AVC por laudo médico ou exame de neuroimagem, constituindo estes os critérios de inclusão. Os critérios de exclusão para este estudo foram indivíduos com outras doenças neurológicas associadas, que estivessem sendo submetidos à TCI como forma de tratamento ou que o tivessem feito há menos de um ano.

A responsável pelo paciente foi orientada quanto ao objetivo do estudo e assinou o Termo de Consentimento Livre e Esclarecido. O participante foi avaliado quanto aos aspectos funcionais com o instrumento Pediatric Motor Activity Log (PMAL) (TAUB; GRIFFIN; 2012), e amplitude de movimento passiva e ativa de todas as articulações dos MMSS por meio de goniometria (MARQUES, 2003).

Quanto ao PMAL trata-se de versão traduzida para este estudo, pelas pesquisadoras responsáveis, o qual consiste em um questionário que foi respondido primeiramente pelo cuidador e logo após, as pesquisadoras juntamente com a mãe do participante realizaram a análise de cada tarefa do questionário resultando na pontuação final (TAUB; GRIFFIN, 2012).

O PMAL questiona se a criança é capaz ou não de realizar determinadas atividades com o membro superior parético. As tarefas avaliadas são: comer com as mãos, pegar pequenos objetos, alimentar-se com uso de garfo ou colher, escovar os dentes, gesticular, colocar o braço na manga de uma blusa, passar a página de um livro, apontar uma imagem, alcançar um objeto acima da cabeça, apertar botão ou tecla, manter-se em equilíbrio em pé, abrir porta, girar uma peça de brinquedo, usar o braço para realizar transferências, tirar os sapatos, tirar as meias, pressionar um objeto, segurar uma pequena bola, jogar bola com as mãos, manipular lápis, empurrar brinquedo de rodas e guardar objetos. A pontuação é dada de acordo com as escalas de frequência e qualidade. Com relação à frequência de uso do membro parético, 0 significa que a criança não usa; 1 usa muito raramente; 2 usa raramente; 3 usa às vezes; 4 sempre usa; 5 usa normalmente. Já a qualidade de uso do membro parético pela criança deve ser classificada em 0 quando não usa; 1 quando os movimentos são muito pobres; 2 para movimentos pobres; 3 para uso moderado do membro; 4 para movimento quase normal; e 5 para execução normal do movimento (TAUB; GRIFFIN, 2012).

A goniometria passiva e ativa foi feita para os movimentos de flexão, extensão, rotação interna e externa, abdução e adução de ombro; flexão, extensão, pronação e supinação de cotovelo; flexão, extensão, desvios radial e ulnar de punho; flexão e extensão de dedos do membro parético.

A criança foi submetida a uma avaliação inicial (AI), antes do início da intervenção; uma avaliação final logo após um mês de intervenção (AFI) e outra após 3 meses do final da intervenção (AFII). As três avaliações foram realizadas na Clínica Escola de Fisioterapia da Universidade São Francisco (CEF-USF). Após a AI, iniciou-se a intervenção com a TCI, três sessões por semana, com duração de três horas cada, ao longo de um mês, sendo duas vezes por semana na CEF-USF e uma vez na residência do paciente. Durante as sessões, antes de conter o membro não afetado, era realizada a preparação da musculatura do membro parético através de mobilização passiva, alongamento passivo, descarga de peso e facilitação 
neuromuscular proprioceptiva. Logo após, a criança teve o membro não afetado imobilizado por faixas de modo que não pudesse ser utilizado nas tarefas propostas, permanecendo junto a seu tronco. O paciente foi estimulado a realizar exercícios fisioterapêuticos, atividades de vida diária, de lazer e escolares em que foram identificadas dificuldades na AI, utilizando materiais da CEF - USF e que os pais trouxeram de casa, quando necessário. Durante a intervenção algumas tarefas como manipular pequenos objetos e jogar boliche com o membro superior parético foram registradas em fotos e vídeos (Figuras 1 e 2).
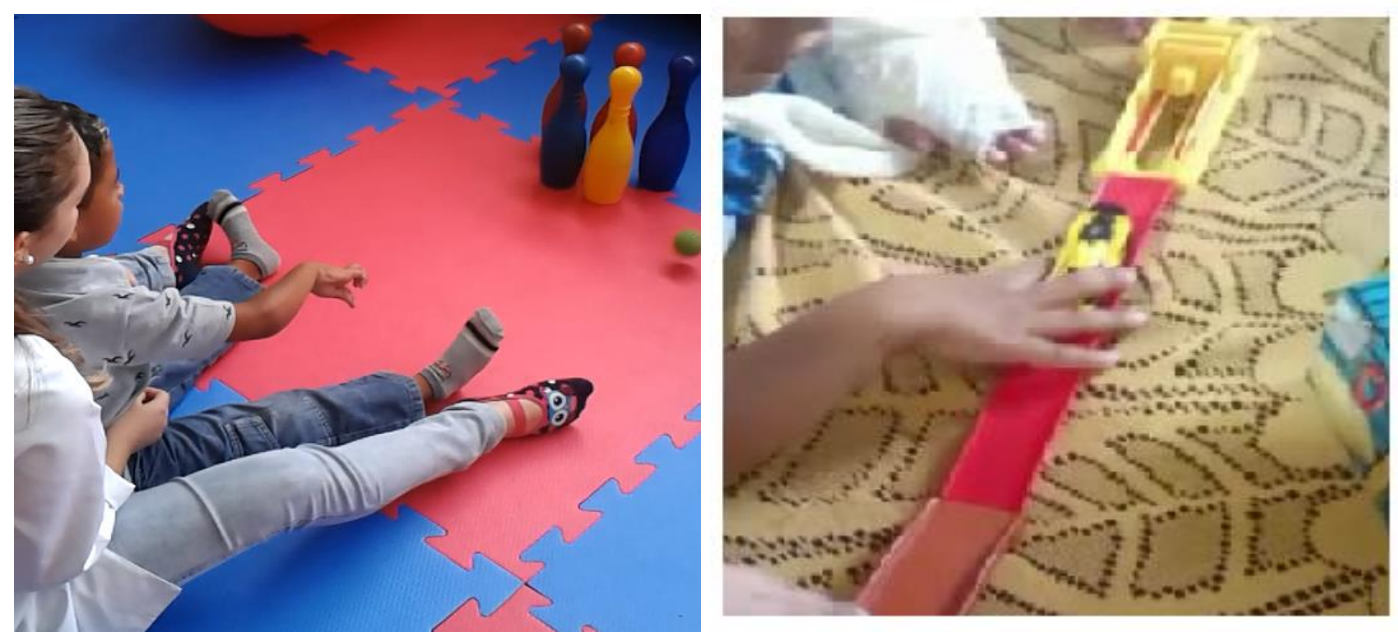

Figuras 1 e 2: Fotos das intervenções realizadas com o paciente, jogando boliche (figura 1) e manipulando pequeno objeto com o membro superior parético (figura 2). (Fonte: autoras)

Para a análise descritiva dos dados, utilizou-se a pontuação obtida no PMAL da avaliação em que a mãe respondeu juntamente com as pesquisadoras; enquanto a versão do PMAL que a mãe respondeu sozinha, foi aproveitada neste estudo como complementação dos resultados, utilizando o relato de suas impressões.

Após análise descritiva geral, foi realizada comparação entre os dados da AI e da AFI para verificar os ganhos imediatos e também da AFII, para verificar se houve manutenção dos efeitos obtidos com o protocolo proposto, em longo prazo de modo que estes serão apresentados a seguir em tabelas e gráficos.

\section{RESULTADOS E DISCUSSÃO}

A busca ativa foi realizada na cidade de Amparo-SP em quatro Unidades de Saúde da Família (USF), um hospital e na Associação de Pais e Amigos dos Excepcionais (APAE). Em Bragança Paulista-SP, em oito USF, no Ambulatório Geral de Especialidades do Hospital Universitário São Francisco, em um Centro de Equoterapia e na APAE e em Socorro-SP somente na APAE. Houve certa resistência por parte de alguns profissionais responsáveis por esses locais, em colaborar com a busca de participantes. Alguns desconheciam a possibilidade da ocorrência de AVC na infância e outros apenas disseram não haver qualquer criança com esse diagnóstico, mesmo sem realizar ou permitir que as pesquisadoras deste estudo realizassem qualquer busca em fichas e prontuários.

Assim, seguindo os critérios de inclusão e exclusão, foi selecionado um participante, do gênero masculino, com 4 anos de idade, com laudo de exame de ressonância magnética de crânio comprovando AVC fetal.

Quanto à avaliação funcional, foi possível observar que, na comparação da AI com a AFI houve melhora na qualidade das seguintes ações: comer com as mãos, alimentar-se com uso de garfo ou colher, colocar o braço na manga de uma blusa, apontar uma imagem, manter- 
se em equilíbrio em pé, girar uma peça de brinquedo e segurar uma pequena bola. A única ação que obteve melhora isolada em frequência foi abrir portas.

Algumas ações tiveram melhora de qualidade e frequência, foram elas: pegar pequenos objetos, gesticular, alcançar um objeto acima da cabeça, apertar botão ou tecla, jogar bola com as mãos, empurrar brinquedo de rodas e guardar objetos. As outras tarefas, conforme pode-se observar na Tabela 1, não tiveram melhora nem em frequência, nem em qualidade.

Tabela 1 - Pontuações obtidas no PMAL em AI, AFI e AFII.

\begin{tabular}{|c|c|c|c|c|c|c|}
\hline \multirow[t]{2}{*}{ Ações } & \multicolumn{3}{|c|}{ Frequência } & \multicolumn{3}{|c|}{ Qualidade } \\
\hline & $\mathrm{Al}$ & $\mathrm{AFI}$ & AF II & $\mathrm{Al}$ & AF I & AF II \\
\hline Comer com as mãos & 0 & 0 & 1 & 1 & 2 & 2 \\
\hline Pegar pequenos objetos & 0 & 3 & 3 & 0 & 3 & 3 \\
\hline Alimentar-se com uso de garfo ou colher & 0 & 0 & 1 & 1 & 2 & 3 \\
\hline Escovar os dentes & 0 & 0 & 0 & 1 & 1 & 1 \\
\hline Gesticular & 0 & 2 & 3 & 2 & 3 & 3 \\
\hline Colocar o braço na manga de uma blusa & 4 & 4 & 4 & 2 & 3 & 3 \\
\hline Passar a página de um livro & 0 & 0 & 1 & 2 & 2 & 4 \\
\hline Apontar uma imagem & 0 & 0 & 3 & 0 & 1 & 2 \\
\hline Alcançar um objeto acima da cabeça & 0 & 1 & 2 & 2 & 4 & 4 \\
\hline Apertar botão ou tecla & 0 & 1 & 2 & \begin{tabular}{|l|l}
2 \\
\end{tabular} & 3 & 3 \\
\hline Manter-se em equilíbrio em pé & 5 & 5 & 5 & 4 & 5 & 5 \\
\hline Abrir portas & 0 & 3 & 3 & 4 & 4 & 4 \\
\hline Girar uma peça de brinquedo & 0 & 0 & 0 & 0 & 1 & 1 \\
\hline Usar o braço para realizar transferências & 1 & 1 & 3 & 2 & 2 & 3 \\
\hline Tirar os sapatos & 0 & 0 & 4 & 0 & 0 & 2 \\
\hline Tirar as meias & 0 & 0 & 0 & 0 & 0 & 0 \\
\hline Pressionar um objeto & 3 & 3 & 3 & 3 & 3 & 3 \\
\hline Segurar uma pequena bola & 3 & 3 & 3 & 2 & 4 & 4 \\
\hline Jogar bola com as mãos & 0 & 3 & 4 & 0 & 4 & 4 \\
\hline Manipular um lápis & 0 & 0 & 0 & 1 & 1 & 1 \\
\hline Empurrar brinquedo de rodas & 0 & 2 & 3 & 2 & 4 & 4 \\
\hline Guardar objetos & 0 & 4 & 4 & 1 & 4 & 4 \\
\hline
\end{tabular}

PMAL: Pediatric Motor Activity Log

AI: Avaliação Inicial

AFI: Avaliação Final I

AFII: Avaliação Final II

(Fonte: Autoras)

Já na comparação da AFI com a AFII foi possível identificar que houve melhora de frequência das ações: comer com as mãos, gesticular, alcançar um objeto acima da cabeça, apertar botão ou tecla, jogar bola com as mãos e empurrar brinquedo de rodas. As ações que tiveram melhora de frequência e qualidade foram: alimentar-se com uso de garfo ou colher, passar a página de um livro, apontar uma imagem, usar o braço para realizar transferências e tirar os sapatos. Nesta comparação, não houve melhora isolada de qualidade das ações. As outras tarefas, conforme pode-se observar na Tabela 1, permaneceram com a mesma pontuação obtida na AI, sendo elas: escovar os dentes, tirar as meias, pressionar um objeto e manipular um lápis.

Com relação ao ganho de amplitude de movimento do membro superior afetado mensurada por meio da goniometria, é possível observar entre a AI e AFI, que houve ganho de ADM passiva de dedos para o movimento de extensão, de punho para o desvio ulnar, de ombro para os movimentos de flexão, adução e abdução, já em comparação da ADM passiva entre a AFI e AFII notou-se ganho para o movimento de rotação interna de ombro somente. 
Tabela 2 - ADM do membro superior direito na AI, AFI e AFII.

\begin{tabular}{|c|c|c|c|c|c|c|}
\hline \multirow[t]{2}{*}{ Movimentos } & \multicolumn{3}{|c|}{ ADM Passiva } & \multicolumn{3}{|c|}{ ADM Ativa } \\
\hline & $\mathrm{Al}$ & AF I & AF II & $\mathrm{Al}$ & AF I & AF II \\
\hline Flexão de dedos & $90^{\circ}$ & $90^{\circ}$ & $90^{\circ}$ & $90^{\circ}$ & $90^{\circ}$ & $90^{\circ}$ \\
\hline Extensão de dedos & $40^{\circ}$ & $50^{\circ}$ & $40^{\circ}$ & $8^{\circ}$ & $8^{\circ}$ & $8^{\circ}$ \\
\hline Flexão de punho & $90^{\circ}$ & $90^{\circ}$ & $90^{\circ}$ & $90^{\circ}$ & $90^{\circ}$ & $90^{\circ}$ \\
\hline Extensão de punho & $60^{\circ}$ & $60^{\circ}$ & $60^{\circ}$ & $26^{\circ}$ & $36^{\circ}$ & $30^{\circ}$ \\
\hline Desvio radial & $20^{\circ}$ & $20^{\circ}$ & $20^{\circ}$ & $16^{\circ}$ & $18^{\circ}$ & $18^{\circ}$ \\
\hline Desvio ulnar & $20^{\circ}$ & $24^{\circ}$ & $24^{\circ}$ & $16^{\circ}$ & $20^{\circ}$ & $16^{\circ}$ \\
\hline Flexão de cotovelo & $130^{\circ}$ & $130^{\circ}$ & $130^{\circ}$ & $122^{\circ}$ & $122^{\circ}$ & $126^{\circ}$ \\
\hline Extensão de cotovelo & $0^{\circ}$ & $\sigma^{\circ}$ & $0^{\circ}$ & $-10^{\circ}$ & $-8^{\circ}$ & $-8^{\circ}$ \\
\hline Pronação & $90^{\circ}$ & $90^{\circ}$ & $90^{\circ}$ & $90^{\circ}$ & $90^{\circ}$ & $90^{\circ}$ \\
\hline Supinação & $42^{\circ}$ & $42^{\circ}$ & $42^{\circ}$ & $20^{\circ}$ & $42^{\circ}$ & $40^{\circ}$ \\
\hline Flexão de ombro & $160^{\circ}$ & $174^{\circ}$ & $170^{\circ}$ & $160^{\circ}$ & $164^{\circ}$ & $160^{\circ}$ \\
\hline Extensão de ombro & $50^{\circ}$ & $50^{\circ}$ & $50^{\circ}$ & $50^{\circ}$ & $50^{\circ}$ & $50^{\circ}$ \\
\hline Rotação interna de ombro & $84^{\circ}$ & $84^{\circ}$ & $90^{\circ}$ & $70^{\circ}$ & $76^{\circ}$ & $80^{\circ}$ \\
\hline Rotação externa de ombro & $90^{\circ}$ & $90^{\circ}$ & $90^{\circ}$ & $62^{\circ}$ & $80^{\circ}$ & $86^{\circ}$ \\
\hline Abdução de ombro & $170^{\circ}$ & $172^{\circ}$ & $172^{\circ}$ & $148^{\circ}$ & $148^{\circ}$ & $148^{\circ}$ \\
\hline Adução de ombro & $40^{\circ}$ & $50^{\circ}$ & $50^{\circ}$ & $36^{\circ}$ & $36^{\circ}$ & $36^{\circ}$ \\
\hline
\end{tabular}

ADM: Amplitude de Movimento

AI: Avaliação Inicial

AFI: Avaliação Final I

AFII: Avaliação Final II

(Fonte: autoras)

No que se refere à ADM ativa, quando comparada a AI e AFI observou-se ganho para extensão de punho, desvio radial, desvio ulnar, extensão de cotovelo, supinação de antebraço, flexão, rotação interna e externa de ombro. Já na comparação da AFI e AFII foi possível identificar ganho de ADM ativa para o movimento de flexão de cotovelo, sendo possível também notar que houve um maior ganho dos movimentos de rotação interna e externa de ombro na AFII em relação a AFI.

Quando comparados os valores da ADM passiva da AFI com AFII, foi possível observar uma redução da amplitude de movimento de extensão de dedos e flexão de ombro, já com relação amplitude ativa de movimento nota-se uma redução da amplitude de movimento de extensão de punho, desvio ulnar de punho, supinação de antebraço e flexão de ombro. As demais amplitudes de movimento, como é possível observar na Tabela 2, não obtiveram ganhos.

O presente estudo utilizou um protocolo de Terapia por Contensão Induzida (TCI) criado e proposto pelas pesquisadoras, após ampla revisão de literatura em que foram encontradas diferentes propostas (GAMBA; CRUZ, 2011; BALEOTTI; GRITTI; et al, 2014; BROL; BORTOLOTO; et al, 2009; MARTINS; SANTOS; et al, 2015). Mesmo os protocolos que utilizaram tempo de aplicação diário menor foi observado melhora significativa na função manual. Por este motivo e pelo fato do participante apresentar idade inferior a maioria dos estudos, optou-se por tempo reduzido das sessões.

Após análise dos resultados obtidos nas três avaliações realizadas foi possível constatar melhora na função manual, visto que houve progresso em 18 das 22 tarefas avaliadas pelo PMAL, sugerindo ganho funcional na realização das AVD’s, atividades escolares e de lazer.

Na comparação entre a AI e AFI houve aumento da pontuação de qualidade maior que de frequência, o que pode ser atribuído ao fato do paciente ter melhorado a percepção quanto 
a sua capacidade em utilizar o membro afetado nas tarefas, embora ainda desse preferência ao membro não afetado para realiza-las.

A melhora em qualidade e frequência pode ser atribuída ao fato de que durante a intervenção eram realizadas repetições de exercícios em que os movimentos estimulados eram semelhantes aos reproduzidos nessas tarefas, facilitando a sua aprendizagem e melhora na execução.

Ainda na comparação da AI com AFI, identificou-se melhora apenas de qualidade em comer com as mãos, alimentar-se com uso de garfo ou colher, colocar o braço na manga de uma blusa, apontar uma imagem, equilibrar-se em pé, girar uma peça de brinquedo e segurar uma pequena bola. Esse achado pode-se justificar pelo fato de serem tarefas funcionais que foram mais treinadas e incentivadas; entretanto, podem ser executadas apenas com um membro superior. Portanto, houve melhora na qualidade, mas não na frequência de uso do membro parético.

As tarefas que não apresentaram melhora da pontuação nem de frequência e nem de qualidade foram escovar os dentes, tirar as meias, pressionar um objeto e manipular lápis. Pode-se justificar este ocorrido pelo fato de serem tarefas que foram menos treinadas na intervenção e menos incentivadas em casa. Além do paciente preferir utilizar o membro não afetado por ser mais rápido e fácil, o que configura principal motivo do desuso.

Resultados semelhantes também foram obtidos no estudo de Baleotti; Gritti; et al (2014), em que foi aplicado um protocolo modificado de TCI em uma criança de 4 anos de idade com hemiparesia espástica decorrente de paralisia cerebral e avaliada também com o PMAL. Houve melhora significativa de qualidade e frequência do uso do membro afetado tanto logo após o tratamento quanto após 6 meses da intervenção, e as tarefas de comer com uso de garfo ou colher, tirar os sapatos e meias não tiveram melhora após a intervenção, provavelmente por serem tarefas que necessitam de maior destreza motora.

Na comparação da AFI com AFII houve manutenção de muitas das tarefas avaliadas, além de melhora de qualidade e frequência de algumas destas, conforme visto na Tabela 1 , demonstrando que o protocolo foi eficaz para manter os ganhos obtidos após a intervenção e promover melhora funcional mesmo após o término. Porém, em algumas tarefas, houve melhora apenas da frequência, o que evidencia o aumento da percepção de sua capacidade de uso do membro afetado mesmo após um período sem intervenção.

O estudo de Brandão; Mancini; et al (2010), demonstrou que um protocolo de TCI modificado melhorou o uso espontâneo do membro superior e o uso do membro nas AVD's, porém, diferente do presente estudo, não observaram ganho quanto à destreza motora.

Outros estudos demonstram que houve manutenção dos ganhos obtidos da função motora mesmo após o término das intervenções através da TCI, sugerindo ganhos motores mesmo após longos prazos após a última intervenção realizada, variando de um mês até um ano (SMITH; DE LUCCA; et al, 2012; SAKZEWSKI; ZIVIANI; et al, 2011; ROSTAMINI; ARRASTOO; et al, 2012 ; SMANIA; AGLIOTI, 2009).

Segundo relatos da mãe, logo após a intervenção, a criança demonstrou melhora da qualidade do uso do membro parético evidenciado em atividades que requeriam alcance, manipulação e preensão de objetos, mas ainda demonstrando clara preferência pelo uso do membro não afetado, confirmando os achados. Após 3 meses da intervenção, na AFII, a mãe relatou aumento do uso do membro afetado principalmente em tarefas bimanuais, o que não era realizado antes da intervenção, demonstrando que mesmo um tempo após a intervenção, ainda houve ganhos.

Tais dados vão ao encontro dos resultados obtidos no estudo de Smania et al (2009) ${ }^{20}$, em que o grupo que recebeu a intervenção fisioterapêutica com o uso da TCI apresentou melhora na função uni e bimanual do membro superior afetado sendo que os ganhos se mantiveram após 4 semanas do término do tratamento. 
Já se sabe que quando a intervenção fisioterapêutica é realizada de forma adequada, o sistema nervoso pode se reorganizar, e isto ocorre devido à plasticidade neural que pode ser definida como o modo que o cérebro codifica experiências e aprende ou reaprende as ações motoras, o que é possível quando existe repetição, intensidade, progressão e transferência da tarefa (KLEIN; JONES, 2008; CARRATÚ; MAZZITELLI; et al, 2012). Além disso, a plasticidade neural tende a ser mais efetiva em crianças, pois com o passar do tempo ocorre redução fisiológica na quantidade de neurônios, o que é chamado de morte neuronal seletiva. Uma criança tem maior número de conexões entre as células neuronais quando comparado as de um adulto, porém, estas conexões são mais fracas que as encontradas em um cérebro maduro, evidenciando que a criança deve receber estímulos neurossensoriais adequados para que conexões importantes sejam mantidas e continuem seu processo de maturação (KLEIN; JONES, 2008; GILLICK; KRACK; et al, 2015).

Segundo Diniz, Abranches et al (2003), o mecanismo de neuroplasticidade mais provável gerado pela TCI é o aumento das conexões sinápticas, induzidas principalmente pelo treinamento repetitivo, podendo até gerar brotamentos neuronais. Souza et al. ${ }^{25}$ relata que as atividades repetitivas que demandam atenção, têm o potencial de facilitar a aprendizagem motora e a neuroplasticidade, tanto na área lesada quanto em áreas não afetadas.

Existem também alguns estudos que comprovaram que o uso forçado do MS parético em treinamento de atividades funcionais pode promover o aumento da representação da área cortical acometida, proporcionando assim a melhora do desempenho motor, otimizando o aprendizado de tarefas e revertendo o desuso aprendido (SOUZA; CONFORTO, 2007).

A TCI, através do uso forçado e do treino de atividades funcionais, com movimentos repetitivos do membro parético, aumenta a representação da área cortical acometida, proporcionando melhor desempenho motor, otimizando o aprendizado e revertendo o desuso aprendido (MENEGHETTI; SILVA, 2010; BARATO; FERNANDES; et al, 2009).

Com relação à avaliação da ADM passiva através de goniometria, notou-se melhora de extensão de dedos, desvio ulnar, flexão, abdução e adução de ombro do membro afetado na comparação da AI com AFI. Houve manutenção de todos na AFII, exceto da extensão de dedos e da flexão de ombro que tiveram piora de $10^{\circ}$ e $4^{\circ}$ respectivamente. Da comparação entre AFI e AFII houve aumento apenas da ADM de rotação interna de ombro e manutenção dos outros movimentos. O aumento da ADM entre a AI e AFI pode ser justificado pelo conjunto de exercícios incluídos no protocolo utilizado, pois antes de iniciar o treino funcional com o membro afetado eram realizadas condutas de preparação, como mobilização passiva, alongamento passivo, facilitação neuromuscular proprioceptiva e descarga de peso em ambos os membros superiores. E as perdas podem ser justificadas pela ausência dessas condutas, já que após o protocolo aplicado, o paciente ficou 3 meses sem qualquer intervenção.

Assim como este, o estudo de Bôas, Fernandes et al (2013), realizava alongamento prévio antes da aplicação de um protocolo de Terapia Virtual como forma de preparar a musculatura do membro a ser estimulado de crianças hemiparéticas por paralisia cerebral, obtendo como resultado ganho funcional, melhora das habilidades e destreza motora.

Já a ADM ativa apresentou melhora nos movimentos de extensão de punho, desvios radial e ulnar, extensão de cotovelo, supinação e flexão de ombro. Houve manutenção de desvio radial e extensão de cotovelo. Na comparação da AFI com AFII houve aumento de $\mathrm{ADM}$ ativa de flexão de cotovelo e rotações interna e externa de ombro. Notou-se redução de $6^{\circ}$ de ADM ativa de extensão de punho, $4^{\circ}$ de desvio ulnar, $2^{\circ}$ de supinação e $4^{\circ}$ de flexão de ombro. Assim, é possível que os aumentos na ADM ativa tenham ocorrido por um real aumento no uso do membro afetado; entretanto, com o término do protocolo, o participante pode ter exercitado menos algumas tarefas mais específicas e, até mesmo pode ter diminuído força muscular, o que pode justificar a perda de ADM ativa. 
Atualmente, pode-se encontrar embasamento na literatura científica comprovando a importância da Fisioterapia para pacientes com lesões neurológicas, confirmando ganhos funcionais através do aumento e manutenção de força muscular e amplitude de movimento. Sabe-se também que o tratamento fisioterapêutico realizado para pacientes neurológicos deve ser contínuo já que estes podem sofrer perdas secundárias às lesões congênitas ou adquiridas (BORELLA; SACHELLI, 2009).

\section{CONCLUSÃO}

Pode-se concluir que a TCI melhorou a função manual em frequência e qualidade do uso do membro parético na maioria das tarefas avaliadas; assim como a ADM passiva e ativa de algumas articulações deste membro do participante. Portanto, a TCI foi eficaz no caso estudado; porém, há necessidade de maior número amostral e utilização de instrumentos de avaliação menos subjetivos para comprovação científica da técnica para pacientes com AVC Infantil.

\section{REFERÊNCIAS}

Baleotti LR, Gritti CC, Silva B.C. Efeitos de um protocolo modificado de terapia por contenção induzida em crianças com paralisia cerebral hemiparética. Rev Ter Ocup. 2014.25: 264-271.

Barato G, Fernandes T, Pacheco M, Bastos VH, Machado S, Mello MP. Cortical plasticity and neurological physical therapy techniques in neuroimage optic. Revista Neurociências. 2009. 17: 342- 8 .

Bôas AV, Fernandes WLM, Silva AM, Silva AT. Efeito da Terapia virtual na Reabilitção Motora do Membro Superior de Crianças Hemiparéticas. Revista Neurociências. 2013. 21: 556- 62 .

Borella MP, Sacchelli T. Os efeitos da prática de atividades motoras sobre a neuroplasticidade. Revista Neurociências. 2009. 17:161- 9.

Brandão MB, Mancini MC, Vaz DV, Melo APP. Adapted version of constraint-induced movement therapy promotes functioning in children with cerebral palsy: a randomized controlled trial. Clin Rehabil. 2010. 24: 639-47.

Brol AM, Bortoloto F, Magagnin NMS. Tratamento de restrição e indução do movimento na reabilitação funcional de pacientes pós acidente vascular encefálico: uma revisão bibliográfica. Rev Fisioterapia em Movimento. 2009. 22: 497-509.

Carratú S, Mazzitelli C, Xavier GF, Sá CSC. Aquisição, Retenção e Transferências de Habilidades Motoras em Crianças Hemiparéticas. Revista Neurociências. 2012. 20: 360- 6.

Deveber G, Kirton A. Therapeutic Approaches and Advances in Pediatric Strokes. The Journal of the American Society for Experimental NeuroTherapeutics. 2006. April; 3: 133142.

Diniz L, Abranches MHS. Neuroplasticidade na terapia de restrição e indução do movimento em pacientes com acidente vascular encefálico. Med Reabilitation. 2003 22: 53-5. 
Gamba RT, Cruz DMC. Efeitos da Terapia por Contenção Induzida em longo prazo em pacientes pós AVC. Revista neurociência. 2011. 19: 735- 40.

Gianlorenço ANL, Kirizawa JM, Faganello FR. Influência da terapia de contenção induzida na função motora do membro superior de indivíduos hemiparéticos. Terapias manuais. 2013. 11: 181-6.

Gillick BT, Krach LE, Feyma T, Rich TL, Moberg K, Meck J, Cassidy J, Kimberley T, Carey JR. Safety of Primed Repetitive Transcranial Magnetic Stimulation and Modified ConstraintInduced Movement Therapy in a Randomized Controlled Trial in Pediatric Hemiparesis. Archives of Physical Medicine and Rehabilitation. 2015. 96: 104-13.

Guimarães IE, Ciasca SM, Ribeiro VL. Neuropsychological evaluation of children after ischemic cerebrovascular disease. Arq Neuropsiquiatr. 2002. 60: 386-389.

Kleim JA, Jones TA. Principles of Experience-Dependent Neural Plasticity: Implications for Rehabilitation After Brain Damage. Journal of Speech Language and Hearing Research. 2008. 51: 225-39.

Leite JMRS, Prado JF. Paralisia cerebral: Aspectos Fisioterapêuticos e Clínicos. Revista Neurociências. 2004. 12: 41-5.

MARQUES AP. Manual de Goniometria. 2a Edição. Barueri. Editora Manole. 2003; Jun; 81.

Martins JS, Santos LF, Castagna LO. Uso da Terapia por Contensão Induzida em indivíduos com paralisia cerebral: uma revisão de literatura. Rev do Departamento de Educação Física e Saúde e do Mestrado em Promoção da Saúde da Universidade de Santa Cruz do Sul. 2015. 16: 214-20.

Matta APC, Galvão KRF, Oliveira BS. Cerebrovascular Disorders in Childhood. Arq Neuropsiquiatr. 2006. Junho; 64: 181-5.

Mekiterian EF, Carvalho WB. Stroke in Children: Acidentes vasculares encefálicos em pediatria. Jornal de Pediatria. 2009. 85: 469-479.

Meneghetti CHZ, Silva JA, Guedes CAV. Constraint-induced movement therapy in chronic stroke: case report. Revista Neurociências. 2010. 23: 18-23.

Palavro BEM, Schuster R C. Efeitos da Terapia de Contenção Induzida Adaptada na Funcionalidade e Qualidade de Vida de Pacientes Hemiparéticos. Revista Fisioterapia e Funcional. 2013. 2: 51-60.

Rodrigues A, Mafalda 1, Fonseca S, Carvalhosa G, Neto AS. Acidente vascular cerebral perinatal: 11 casos clínicos. Acta Pediátrica Portuguesa. 2015. Abril; 46: 24-7.

Rostamini HR, Arastoo AA, Nejad SJ, Mahany MK, Malamiri R, Goharpey S. Effects of modified constraint-induced movement therapy in virtual environment on upper-limb function in children with spastic hemiparetic cerebral palsy: A randomized controlled trial. Neuro Rehabil. 2012. 31: 357-65. 
Rotta NT, Silva AR, Ohlweiler FLF, Belarmino I, Fonteles E, Ranzan J, Rodriguez VR, Martins OJR, Cerebrovascular diasease in pediatric patients. Arq Neuropsiquiatr. 2002. 60: 959-963.

Sakzewski L, Ziviani J, Abbott DF, Macdonel RA, Jacson GD Boyd RN. Equivalent retention of gains at 1 year after training with constraint-induced or bimanual therapy in children with unilateral cerebral palsy. Neurorehabil Neural Repair. 2011. 25: 664-725.

Silva L A, Tamashiro V, Assis RD. Terapia por contenção induzida: revisão de ensaios clínicos. Fisioterapia do movimento. 2010. 23: 153-9.

Smania N, Aglioti SM, Cosentino A, Camin M, Gandolfi M, Tinazzi M, Fiaschi A, Faccioli S. A modified Constraint-Induced Movement Therapy (CIT) program improves paretic arm use and function in children with cerebral palsy. Eur J Phys Rehabil Med. 2009. 45: 493-500.

Smith J, De Lucca SC, Steverson R, Ramey SL. Multicenter randomized controlled trial of pediatric constraint-induced movement therapy: 6-month follow-up. J.Occup Ther. 2012. 66: $15-23$.

Souza WC, Conforto AB, André C. Terapia de restrição e indução do movimento. Fisioterapia Brasil. 2007. 8: 64-8.

Taub E, Griffin A, Uswatte G. Pediatric Upper Extremity Motor Activity Log - Revised (PMAL) Manual. University of Alabama at Birmingham and Children's Hospital of Alabama. 2012. 